PROCEEDINGS OF THE AMERICAN MATHEMATICAL SOCIETY

Volume 124, Number 10, October 1996

\title{
A SIMPLE PROOF OF SINGER'S REPRESENTATION THEOREM
}

\author{
WOLFGANG HENSGEN
}

(Communicated by Dale E. Alspach)

\begin{abstract}
Let $\Omega$ be a compact Hausdorff space and $X$ a Banach space. Singer's theorem states that under the dual pairing $(f, m) \mapsto \int\langle f, d m\rangle$, the dual space of $C(\Omega ; X)$ is isometric to $\operatorname{rcabv}\left(\Omega ; X^{\prime}\right)$. Using the Hahn-Banach theorem and the (scalar) Riesz representation theorem, a proof of Singer's theorem is given which appears to be simpler than the proofs supplied earlier by Singer $(1957,1959)$ and Dinculeanu $(1959,1967)$.
\end{abstract}

The notation of the abstract is supposed to be self-explanatory. A Borel measure $m \in \operatorname{cabv}(\Omega ; X)$ is called regular $(m \in r c a b v)$ iff its variation $|m|$ is regular. (As a corollary of our approach it is shown below that this is equivalent to the weakest possible notion of regularity.)

Proof of Singer's representation theorem. a) Let $m \in \operatorname{cabv}\left(\Omega ; X^{\prime}\right)$; then routine verifications show that $\varphi_{m}(f):=\int\langle f, d m\rangle$ defines a functional $\varphi_{m} \in C(\Omega ; X)^{\prime}$ of norm $\left\|\varphi_{m}\right\| \leq\|m\|(=|m|(\Omega))$. Under the additional requirement that $\langle x, m\rangle$ be regular $\forall x \in X, m$ is uniquely determined by $\varphi_{m}$, by the uniqueness part of the (scalar) Riesz theorem.

b) Conversely, let $\varphi \in C(\Omega ; X)^{\prime}$ be given. Equip the unit ball $B_{X^{\prime}}$ with the weak* topology and consider the isometric embedding $C(\Omega ; X) \hookrightarrow C\left(\Omega \times B_{X^{\prime}}\right)$ sending $f$ into the scalar function $\left(\omega, x^{\prime}\right) \mapsto\left\langle f(\omega), x^{\prime}\right\rangle$. The Hahn-Banach theorem combined with the Riesz representation theorem [R, 6.19] produces a complex regular Borel measure $\nu$ on $\Omega \times B_{X^{\prime}}$ satisfying $\|\nu\|=\|\varphi\|$ and $\forall f \in C(\Omega ; X): \varphi(f)=$ $\int_{\Omega \times B_{X^{\prime}}}\left\langle f(\omega), x^{\prime}\right\rangle \nu\left(d \omega, d x^{\prime}\right)$. In particular, $\forall u \in C(\Omega) \forall x \in X$ :

$$
\varphi(u \otimes x)=\int_{\Omega \times B_{X^{\prime}}} u(\omega)\left\langle x, x^{\prime}\right\rangle \nu\left(d \omega, d x^{\prime}\right)=\int_{\Omega} u d \mu_{x}
$$

for this Borel measure $\mu_{x}$ on $\Omega$ :

$$
\mu_{x}(A):=\int_{\Omega \times B_{X^{\prime}}} \mathbf{1}_{A}(\omega)\left\langle x, x^{\prime}\right\rangle \nu\left(d \omega, d x^{\prime}\right) .
$$

Received by the editors April 21, 1995.

1991 Mathematics Subject Classification. Primary 46E15, 46E40.

Key words and phrases. Vector-valued continuous functions, regular vector measures.

(C)1996 American Mathematical Society 
Obviously

$$
\begin{aligned}
\left|\mu_{x}(A)\right| & \leq\|x\| \int_{\Omega \times B_{X^{\prime}}} \mathbf{1}_{A}(\omega)|\nu|\left(d \omega, d x^{\prime}\right) \\
& =\|x\| \int_{\Omega} \mathbf{1}_{A}(\omega) \nu_{1}(d \omega)=\|x\| \nu_{1}(A),
\end{aligned}
$$

where $\nu_{1}:=|\nu| \circ \operatorname{pr}_{1}^{-1}$ is a positive regular Borel measure on $\Omega$, as is easily verified. Defining, for Borel sets $A \subset \Omega$ and $x \in X, m(A)(x):=\mu_{x}(A)$, we have immediately $m(A) \in X^{\prime},\|m(A)\| \leq \nu_{1}(A)$, hence $|m| \leq \nu_{1}$ so that $m \in \operatorname{rcabv}\left(\Omega ; X^{\prime}\right)$ and $\|m\| \leq \nu_{1}(\Omega)=\|\nu\|=\|\varphi\|$. By construction, $m$ represents $\varphi$ on $C(\Omega) \otimes X$ which is dense in $C(\Omega ; X)$ [DU, p.225]. Invoke a) to conclude that $m$ represents $\varphi$ on $C(\Omega ; X)$.

The following corollary is proved directly in [S1, Lemma 3].

Corollary. For $m \in \operatorname{cabv}(\Omega ; Y)$, the following are equivalent:

$1^{\circ}|m|$ is regular.

$2^{\circ}\left\langle m, y^{\prime}\right\rangle$ is regular $\forall y^{\prime} \in X$, a norming subspace of $Y^{\prime}$.

Proof. To prove $2^{\circ} \Rightarrow 1^{\circ}$, we can assume $Y=X^{\prime}$. Suppose $\langle x, m\rangle$ is regular $\forall x \in X$. The functional $\varphi_{m} \in C(\Omega ; X)^{\prime}$ is represented by an $m^{\prime} \in \operatorname{rcabv}\left(\Omega ; X^{\prime}\right)$ which implies $m=m^{\prime}$ as noted in a) above.

\section{REFERENCES}

[DU] J. Diestel, J.J. Uhl, Jr., Vector Measures, AMS, Providence, 1977 (Math. Surveys 15). MR 56: 11216

[D1] N. Dinculeanu, Sur la représentation intégrale des certaines opérations linéaires. III, Proc. AMS 10 (1959), 59-68. MR 21:2909

[D2] N. Dinculeanu, Vector Measures, Pergamon Press, Oxford etc., 1967. MR 34:6011b

[R] W. Rudin, Real and Complex Analysis, 3rd ed., McGraw Hill, New York etc., 1987. MR 88k:00002

[S1] I. Singer, Linear functionals on the space of continuous mappings of a compact Hausdorff space into a Banach space (in Russian), Rev. Roum. Math. Pures Appl. 2 (1957), 301-315. MR 20:3445

[S2] I. Singer, Sur les applications linéaires intégrales des espaces de fonctions continues. I, Rev. Roum. Math. Pures Appl. 4 (1959), 391-401. MR 22:5883

NWF I - Mathematik, Universität Regensburg, D- 93040 Regensburg, Germany

E-mail address: wolfgang.hensgen@mathematik.uni-regensburg.de 